\title{
Evidence of Acute Mycoplasma Infection in a Patient with Incomplete and Atypical Kawasaki Disease: A Case Report
}

\author{
M. Ebrahim, ${ }^{1}$ M. Gabay, ${ }^{1,2,3}$ and R. F. Rivas-Chacon ${ }^{3}$ \\ ${ }^{1}$ Miami Children's Hospital, Miami, FL 33155, USA \\ ${ }^{2}$ Department of Pediatrics, Nova Southeastern University, Fort Lauderdale-Davie, FL 33314-7796, USA \\ ${ }^{3}$ Department of Rheumatology, University of Miami, Miami, FL 33136, USA
}

Correspondence should be addressed to M. Ebrahim, mohdi84@gmail.com

Received 30 August 2011; Revised 31 October 2011; Accepted 1 November 2011

Academic Editor: C. G. M. Kallenberg

Copyright (c) 2011 M. Ebrahim et al. This is an open access article distributed under the Creative Commons Attribution License, which permits unrestricted use, distribution, and reproduction in any medium, provided the original work is properly cited.

\begin{abstract}
The etiology of Kawasaki disease remains unknown despite extensive studies. Some researchers suggest that it is caused by an infectious agent. This is a case report where a patient with incomplete Kawasaki disease was found to have evidence compatible with acute Mycoplasma pneumoniae infection. This is one of the several case reports linking Mycoplasma pneumoniae to Kawasaki disease as a possible trigger. This is perhaps due to a superantigen or is mediated by some other mechanism. Accurate and timely testing for Mycoplasma infections is difficult and has its limitations. Despite this, Mycoplasma pneumoniae should be considered in the differential and workup for Kawasaki disease.
\end{abstract}

\section{Case}

A previously healthy, fully vaccinated 12 -year-old Hispanic male presented with a 6-day history of high-grade and intermittent daily fever (up to $105^{\circ} \mathrm{F}$ ) upon admission. His initial symptoms were sore throat, nausea, and vomiting in addition to fever. Three days prior to admission, the patient was seen in a pediatric emergency department and discharged with a diagnosis of viral illness. The fever persisted, and he became more ill appearing with complaints of weakness, malaise, and myalgias, and a faint generalized, nonpruritic rash began to appear. There was no history of joint involvement, sick contacts, recent travel, or exposures other than a family dog. Physical examination at the time of admission revealed a tachycardic (heart rate of 118 beats/min), tachypneic (respiratory rate of $20 / \mathrm{min})$, febrile $\left(101.5^{\circ} \mathrm{F}\right)$, ill-appearing patient with normal blood pressure (121/75). An erythematous macular rash was present on the face, abdomen, and extremities. $\mathrm{He}$ had conjunctival injection and pharyngeal erythema without observed oral ulcers. Small bilateral cervical lymphadenopathy were palpated and measured to be less than $1.5 \mathrm{~cm}$ in size. There was no associated edema, erythema, or desquamation of the hands or feet.
Laboratory workup was initiated for suspected infectious or rheumatologic causes. Abnormal laboratory findings included a leukocytosis $\left(17 \times 10^{3} / \mathrm{mm}^{3}\right)$ with predominant neutrophils and a normocytic, normochromic anemia $(12.2 \mathrm{mg} / \mathrm{dL})$. Complete blood count showed an initial thrombocytopenia $\left(143 \times 10^{3} / \mathrm{mm}^{3}\right)$ that was later followed by thrombocytosis $\left(398 \times 10^{3} / \mathrm{mm}^{3}\right)$. In addition, analysis revealed an elevated CRP $(14.2 \mathrm{mg} / \mathrm{L})$, ESR $(64 \mathrm{~mm} / \mathrm{h})$, and hypoalbuminemia ( $2.5 \mathrm{~g} / \mathrm{dL})$. Mycoplasma pneumoniae IgM serology was positive (Mycoplasma IgM titer $\geq 1.10$ ). Chest $\mathrm{X}$-ray showed faint bilateral interstitial markings without lung consolidation or collapse. Comprehensive viral studies and cultures from the throat, urine, and blood were negative. The remainder of the initial workup, which included electrolytes, urinalysis, renal function test, and lupus analyzer, was within normal range.

A diagnosis of viral syndrome was initially suspected, although a course of azithromycin $(10 \mathrm{mg} / \mathrm{kg} /$ day $)$ was started. Acute Mycoplasma pneumoniae infection was made after the Mycoplasma IgM serology came back positive. Subsequently, the possibility of Incomplete Kawasaki disease was entertained given the patient's presentation. This was later confirmed on day 1 of hospitalization by an echocardiogram 
TABLE 1: First hospital admission inpatient course.

\begin{tabular}{|c|c|c|c|c|c|c|}
\hline Symptoms/signs & $\begin{array}{c}\text { Day }-3 \\
\text { Vomiting, } \mathrm{ST}^{* 1} \\
\text { fever, malaise }\end{array}$ & $\begin{array}{c}\text { Day } 0 \\
\text { Fever/rash }\end{array}$ & $\begin{array}{c}\text { Day } 1 \\
\text { Fever/rash }\end{array}$ & $\begin{array}{c}\text { Day } 4 \\
\text { Afebrile }\end{array}$ & $\begin{array}{l}\text { Day } 6 \\
\text { Fever }\end{array}$ & $\begin{array}{c}\text { Day } 9 \\
\text { Afebrile/DC*7 }\end{array}$ \\
\hline WBC count, $\times 10^{3}$ cells $/ \mu \mathrm{L}$ & 7.7 & 10.4 & 9.3 & 16.8 & & \\
\hline Hemoglobin, $\mathrm{g} / \mathrm{dL}$ & 12.2 & 12.4 & 10.4 & 8.9 & & \\
\hline Platelet count, $\times 10^{3}$ platelets $/ \mu \mathrm{L}$ & 147 & 243 & 256 & 398 & & \\
\hline $\mathrm{ESR}, \mathrm{mm} / \mathrm{h}$ & & 64 & & 145 & 92 & \\
\hline CRP, mg/dL & & 14.2 & 10.1 & 7.4 & 4.3 & \\
\hline Mycoplasma IgM & & Positive & & & & \\
\hline Other & $\begin{array}{c}\mathrm{RST} \mathrm{Neg}{ }^{* 2} / \mathrm{MST} \\
\mathrm{Neg}^{* 3}\end{array}$ & Albumin 2.5 & Bld cx-neg*4 & Bld cx-neg & & \\
\hline Echo & & & Dilatation of RCA*5 & & New ectasia & \\
\hline Treatment & Tylenol & Azithromycin & IVIG and $\mathrm{ASA}^{* 6}$ & 2nd IVIG/ASA & Prednisone/ASA & $\begin{array}{l}\text { ASA and } \\
\text { prednisone } \\
\text { tapering }\end{array}$ \\
\hline
\end{tabular}

showing mild dilatation of right coronary artery. The patient was immediately started on an IVIG $(2 \mathrm{gm} / \mathrm{kg})$ infusion and high-dose aspirin $(20 \mathrm{mg} / \mathrm{kg} /$ dose every $6 \mathrm{hrs})$.

The patient continued to spike fevers for three days after IVIG infusion. A second dose of $1 \mathrm{gm} / \mathrm{kg}$ IVIG infusion was given, but the inflammatory markers remained elevated and the patient continued to spike fevers throughout. A repeat echocardiogram on day 6 of hospitalization showed progression of the disease with the addition of left main and proximal left anterior descending artery ectasia. Because of the apparent progression of the disease, pulse therapy with IV methylprednisolone $(30 \mathrm{mg} / \mathrm{kg})$ was administered. The patient's symptoms improved significantly with resolution of the fever. The patient was discharged on day 9 of hospitalization with aspirin and a prednisone tapering regimen (Table 1).

Five days after discharge from the hospital, the patient was readmitted due to recurrence of the fever, malaise, and generalized erythematous rash without any new attributable exposure. Inflammatory markers were elevated, but improved when compared to previous results. Prior discharge medications, aspirin and prednisone, were continued, and an infliximab infusion $(5 \mathrm{mg} / \mathrm{kg})$ was initiated. The patient remained febrile, and a second IV methylprednisolone pulse dose $(30 \mathrm{mg} / \mathrm{kg})$ was given after which the patient defervesced and improved. The patient was discharged home to continue a prednisone tapering and aspirin regimen (Table 2).

The patient was then followed in the outpatient setting and has had no recurrence of symptoms. A repeat echocardiogram 10 days after discharge showed the diameter of the right coronary artery to be in the upper limits of normal, but the left main and descending arteries were more dilated and prominent. A one-month follow-up echocardiogram showed complete resolution of the coronary arteries. Repeat Mycoplasma IgM serology drawn ten months after discharge was negative (Mycoplasma IgM titer $\leq 0.90$ ).

\section{Discussion}

Kawasaki disease (KD) is an acute, self-limiting vasculitis of unknown etiology that usually occurs in childhood between the ages of two to five years old [1]. When untreated, 15 to $25 \%$ of patients develop coronary artery aneurysms [2]. It is diagnosed according to the clinical criteria developed by Kawasaki [3], as shown in Table 3. Some patients do not fulfill the clinical criteria for classic KD and are diagnosed based on echocardiogram findings. Such patients are diagnosed with "incomplete" KD. Such patients are usually at extreme of ages and are at more risk for developing coronary artery disease. They may also have unusual findings and manifestations such as thrombocytopenia and hypoalbuminemia.

Because of the prolonged history of unexplained fever, rash, bilateral conjunctival injection, and pharyngeal erythema, incomplete Kawasaki disease was considered in the case patient. Bilateral lymphadenopathy was present but no cervical lymph node measured $1.5 \mathrm{~cm}$ or greater. The patient presented with three out of the five principal diagnostic criteria along with prolonged fever characteristic of classic Kawasaki disease (Table 3). The diagnosis was confirmed sonographically with involvement of the coronary arteries.

Many characteristics of Kawasaki disease suggest that it is caused by an infectious process such as its self-limited, generally nonrecurring nature, fever, and localized outbreaks [2]. It is hypothesized that the vasculitis in $\mathrm{KD}$ may be triggered by an immune response to a superantigen (SAg) from an infectious pathogen in those who are genetically susceptible [4]. Numerous studies showed a skewed T-cell repertoire in KD patients, and animal models have demonstrated hallmarks of superantigen-mediated response. Many of the infectious agents linked to KD such as Staphylococci species share the presence of superantigenic activity [5].

There is limited evidence that Mycoplasma species might be a trigger pathogen, or at least one of them. It has been reported on several occasions that MP infection preceded 
TABLE 2: Second admission inpatient hospital course.

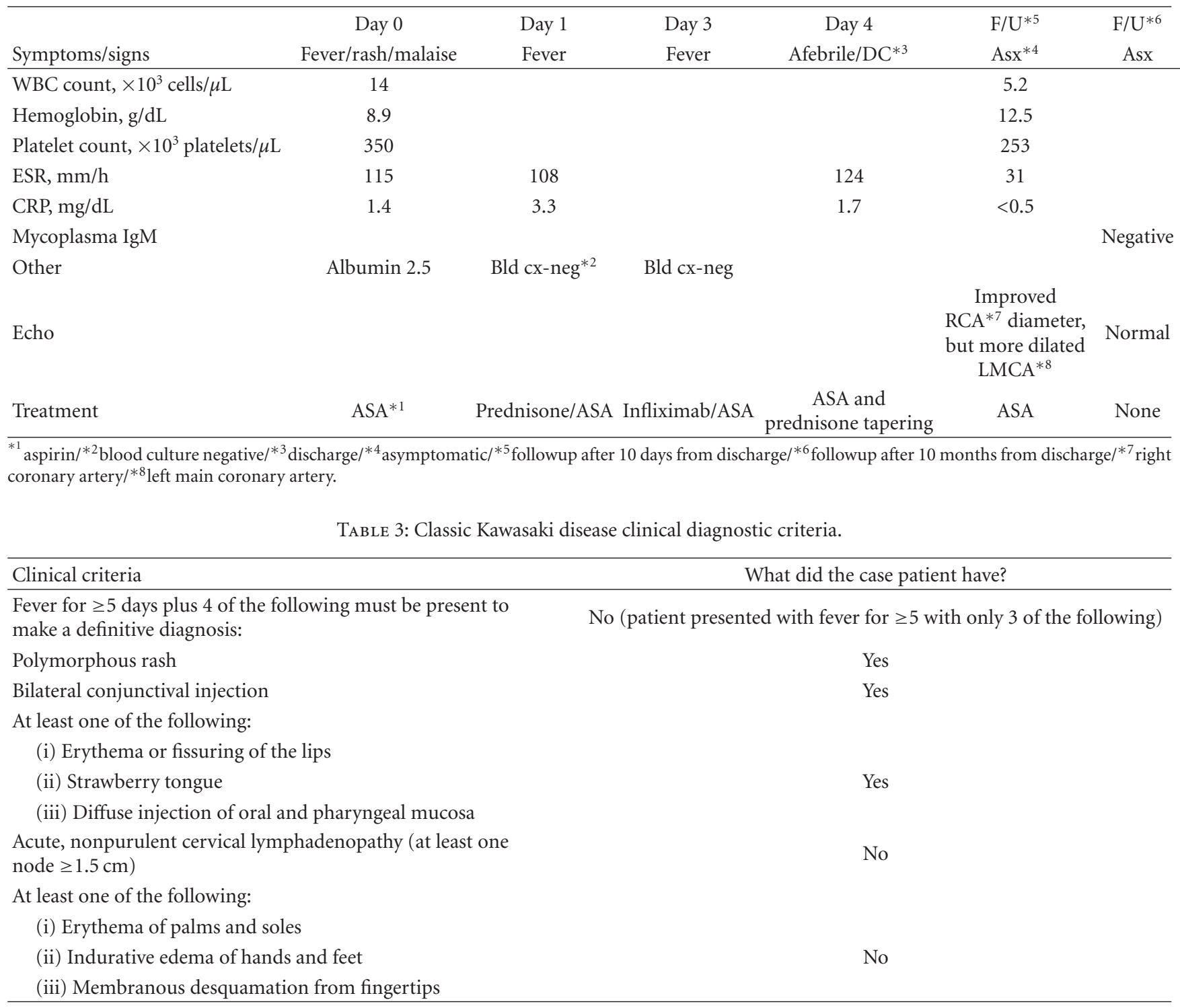

classic KD [6]. MP has been reported to cause cutaneous vasculitis by immune-complex-mediated mechanisms [7]. Another species of the Mycoplasma genome, Mycoplasma arthritidis, has been shown to produce a superantigen suggesting the possibility that other Mycoplasma species may do likewise [8].

The patient had serologic evidence of acute MP infection. To the best knowledge of the writers, this is the first case report of MP infection, or at least evidence of acute infection, followed by incomplete KD.

Diagnosing MP is a difficult task, especially in the face of Kawasaki disease. It is hampered by lack of standardized, rapid, and specific methods. Laboratory tests for MP include culture, serology, and PCR. There is a high ratio of asymptomatic respiratory carriers, and because of continued carriage of the organism after infection and treatment, PCR and cultures from respiratory tract specimens carry the risk of de- tecting healthy carriers. In recent years, the diagnosis of MP has relied mostly on serological methods.

Given the simplicity and benign treatment for MP infection, many clinicians use single-point Mycoplasma IgM serology testing to determine treatment instead of the "gold standard" paired sera conversion test. IgM antibodies are usually produced acutely, and raised IgM levels indicate acute infection. However, it was observed that elevated levels of Mycoplasma IgM might persist for several years raising concern for false-positive results [9]. When only one is positive, antibiotics are generally given as treatment, however, a second sample is ideally required to confirm. In this patient, repeat anti-Mycoplasma level IgM test ten months after initial diagnosis was negative favoring a true-positive initial test.

MP infection can present similarly to KD with prolonged fever, rash, conjunctivitis, and lymphadenopathy, and certain 
species of Mycoplasma genome are known to form superantigen. In this patient, initial Mycoplasma IgM titer at presentation was positive and repeat Mycoplasma IgM titer serology was negative, favoring a true positive test at initial KD presentation. To elucidate a true relationship, if any, between Mycoplasma infection and KD, Mycoplasma testing should be considered in patients found to have KD when clinically suspected which may ultimately affect severity of disease, prognosis, and treatment. The type of testing to pursue to determine acute Mycoplasma infection in the face of $\mathrm{KD}$, however, remains a dilemma.

\section{Disclosure}

There is no source of grant or industrial financial support.

\section{References}

[1] K. A. Taubert, A. H. Rowley, and S. T. Shulman, "Seven-year national survey of Kawasaki disease and acute rheumatic fever," Pediatric Infectious Disease Journal, vol. 13, no. 8, pp. 704-708, 1994.

[2] J. W. Newburger, M. Takahashi, M. A. Gerber et al., "Diagnosis, treatment, and long-term management of Kawasaki disease: a statement for health professionals from the committee on rheumatic fever, endocarditis, and Kawasaki disease, council on cardiovascular disease in the young, American heart association," Pediatrics, vol. 114, no. 6, pp. 1708-1733, 2004.

[3] T. Kawasaki, "Acute febrile mucocutaneous syndrome with lymphoid involvement with specific desquamation of the fingers and toes in children," Japanese Journal of Allergy, vol. 16, pp. 178-222, 1967.

[4] R. M. Kliegman, R. E. Behrman, H. B. Jenson, and B. F. Stanton, Nelson Textbook of Pediatrics, Saunders Elsevier, 2007.

[5] K. Matsubara and T. Fukaya, "The role of superantigens of group A Streptococcus and Staphylococcus aureus in Kawasaki disease," Current Opinion in Infectious Diseases, vol. 20, no. 3, pp. 298-303, 2007.

[6] M. N. Lee, J. H. Cha, H. M. Ahn et al., "Mycoplasma pneumoniae infection in patients with Kawasaki disease," Korean Journal of Pediatrics, vol. 54, no. 3, pp. 122-126, 2011.

[7] F. Greco, A. Sorge, V. Salvo, and G. Sorge, "Cutaneous vasculitis associated with mycoplasma pneumoniae infection: case report and literature review," Clinical Pediatrics, vol. 46, no. 5, pp. 451453, 2007.

[8] M. T. Shio, M. Olivier, S. Jancar, and F. Ribeiro-Dias, "Crucial cytokine interactions in nitric oxide production induced by Mycoplasma arthritidis superantigen," Microbes and Infection, vol. 10, no. 14-15, pp. 1543-1551, 2008.

[9] B. W. Eun, N. H. Kim, E. H. Choi, and H. J. Lee, "Mycoplasma pneumoniae in Korean children: the epidemiology of pneumonia over an 18-year period," Journal of Infection, vol. 56, no. 5, pp. 326-331, 2008. 


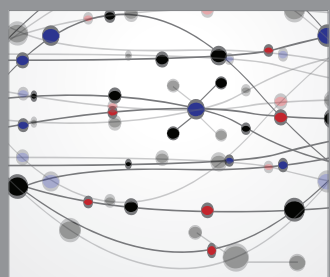

The Scientific World Journal
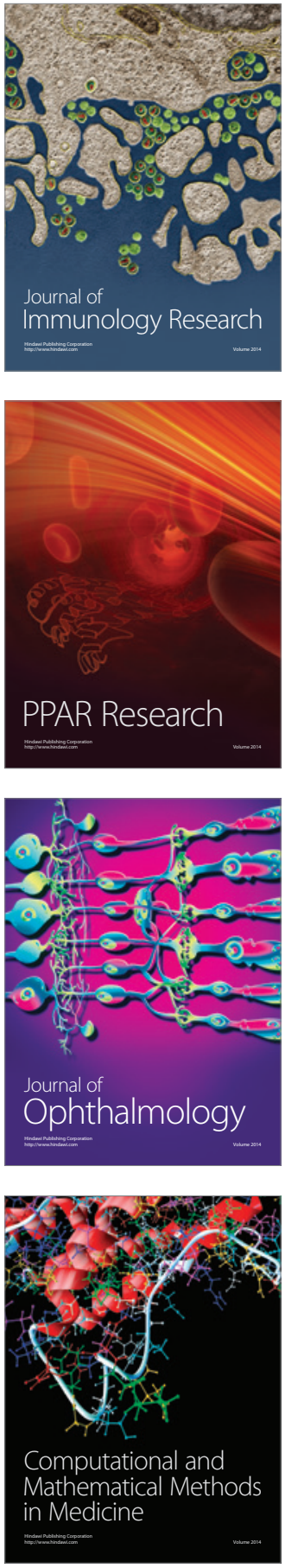

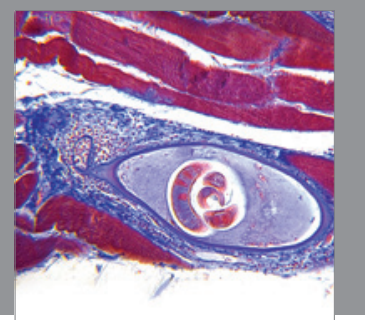

Gastroenterology

Research and Practice
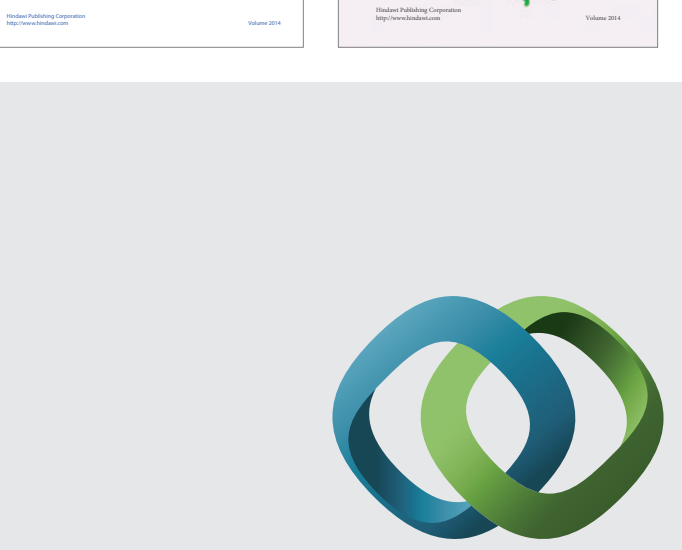

\section{Hindawi}

Submit your manuscripts at

http://www.hindawi.com
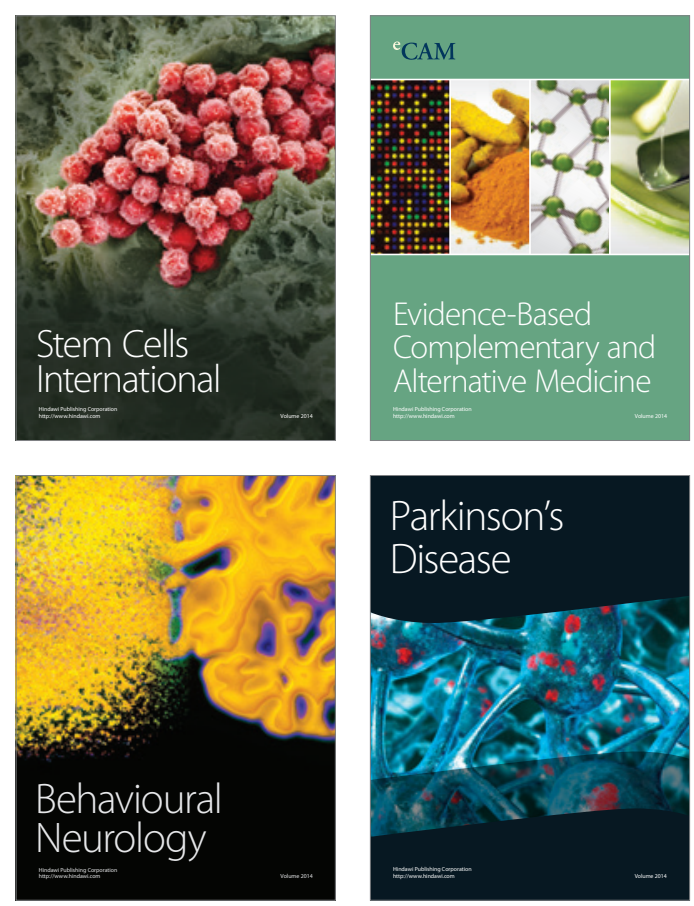

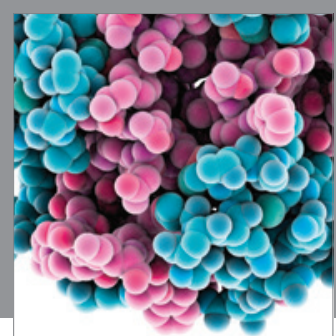

Journal of
Diabetes Research

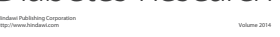

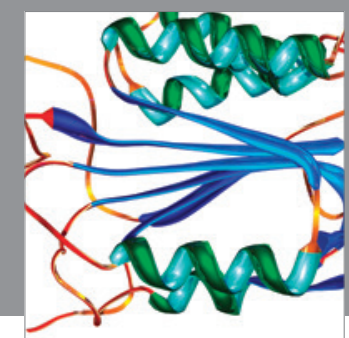

Disease Markers
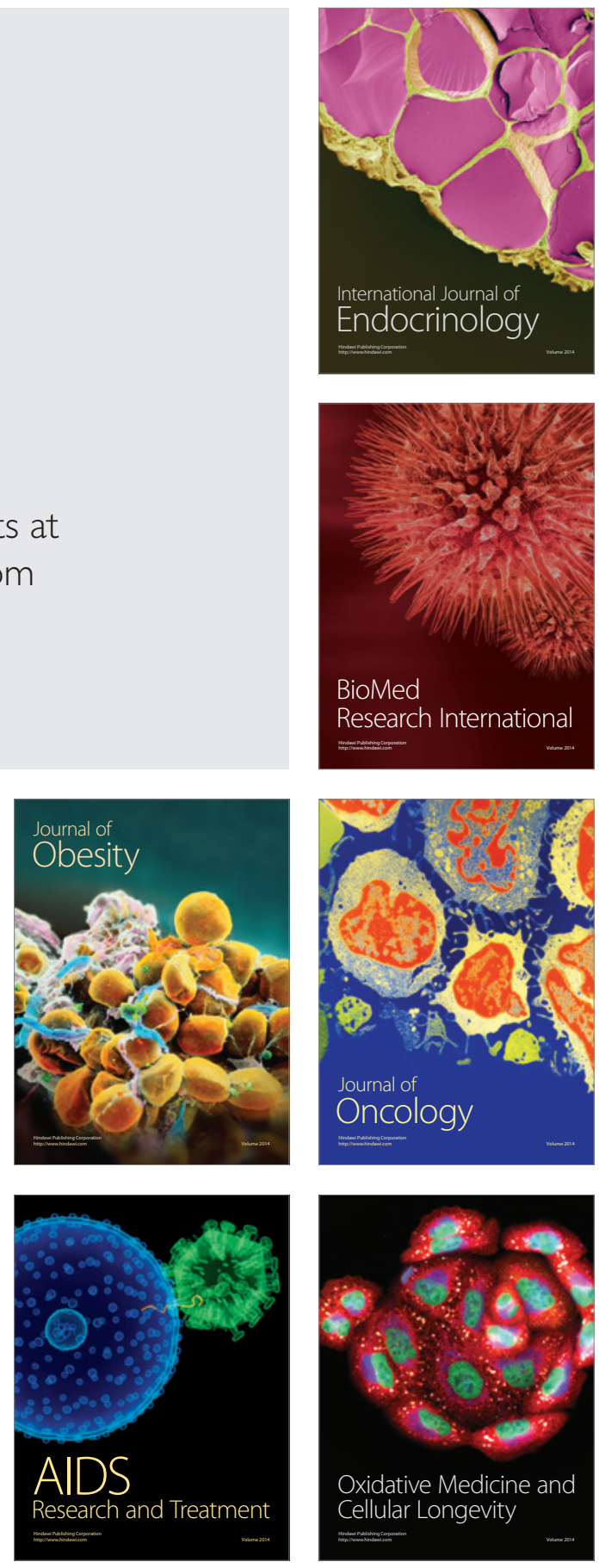